\title{
Structure and Synthesis of 6-(Substituted-imidazol-1-yl)purines: Versatile Substrates for Regiospecific Alkylation and Glycosylation at N9
}

\author{
Minghong Zhong, Ireneusz Nowak, John F. Cannon, and Morris J. Robins* \\ Department of Chemistry and Biochemistry, Brigham Young University, Provo, Utah 84602- \\ 5700 \\ morris_robins@byu.edu
}

\section{Supporting Information (1)}

Pages S2-S8: Experimental procedures and synthesis of compounds $\mathbf{3 b}-\mathbf{3 d}$ and $\mathbf{8 b}-\mathbf{8} \mathbf{e}$.

Pages S9-S13: X-Ray crystal structures of compounds B (Fig. 1), 8c (Scheme 2), D-F (Fig. 2).

Pages S14-S28: ${ }^{13} \mathrm{C}$ NMR spectra of 2, 3a-d, 5, 8a-e, 13a, 13b, 13e, 16a.

${ }^{*}$ Corresponding author. Telephone: (801) 422-6272; Fax: (801) 422-0153. 
Uncorrected melting points were determined with a capillary tube apparatus. UV spectra were determined with solutions in $\mathrm{MeOH} .{ }^{1} \mathrm{H}$ NMR spectra (300 or $500 \mathrm{MHz}$ ) were obtained with $\mathrm{CDCl}_{3}$ or DMSO- $d_{6}$ solutions $\left(\mathrm{Me}_{4} \mathrm{Si}\right.$ internal). Observed multiplicities are within quotation marks for peaks that showed other than first-order splitting. ${ }^{13} \mathrm{C}$ NMR spectra (75 or $\left.125 \mathrm{MHz}\right)$ were obtained in $\mathrm{CDCl}_{3}$ (77.4 ppm internal) or DMSO- $d_{6}(40.5 \mathrm{ppm}$ internal) solutions. Mass spectra (CI, EI, FAB) at high resolution (HRMS) were determined with FAB (glycerol or thioglycerol matrix) unless otherwise noted $\left(\mathrm{CH}_{4}\right.$ was used for $\left.\mathrm{CI}\right)$. Reagent grade chemicals were used. $\mathrm{CH}_{2} \mathrm{Cl}_{2}, \mathrm{CH}_{3} \mathrm{CN}$, DMA, DMF, DCE, pyridine, and toluene were dried by reflux over $\mathrm{CaH}_{2}$ and distilled. Volatile materials were flash evaporated at $\leq 35{ }^{\circ} \mathrm{C}$ under house vacuum $(\sim 12$ $\mathrm{mm} \mathrm{Hg}$ ) or with a mechanical oil pump (in vacuo). TLC was performed with $\mathrm{EtOAc} / \mathrm{iPrOH} / \mathrm{H}_{2} \mathrm{O}$ (4:1:2, upper layer), $\mathrm{MeOH} / \mathrm{CH}_{2} \mathrm{Cl}_{2}$ (ratios of 1:3 to 1:99, v/v), or EtOAc/hexanes (ratios of $85: 15$ to $3: 7, \mathrm{v} / \mathrm{v}$ ) for polar to nonpolar compounds, respectively. Dowex $1 \times 2\left[\mathrm{OH}^{-}\right]$was generated by washing the $\mathrm{Cl}^{-}$form with $1 \mathrm{M} \mathrm{NaOH} / \mathrm{H}_{2} \mathrm{O}$ until no chloride was detected in the eluate upon acidification $\left(\mathrm{HNO}_{3}\right)$ and addition of $\mathrm{AgNO}_{3}$. Solid products were dried in vacuo over $\mathrm{P}_{2} \mathrm{O}_{5}$ for $\geq 1$ day, and stable compounds were dried at elevated temperatures. Method $A^{1}$ usually produced 2-alkylimidazoles in good yields as reported. However, dialkylation (C2 and N3) can make separation of the product from the dialkylated by-product difficult. Complete removal of the reported $\mathrm{N}$-protecting group also was troublesome in some cases. Method $B^{2}$ worked well for volatile aldehydes. Products with up to $90 \%$ purity $\left({ }^{1} \mathrm{H}\right.$ NMR) were obtained beginning with certain aliphatic aldehydes with low boiling points.

\footnotetext{
${ }^{1}$ Katritzky, A. R.; Rewcastle, G. W.; Fan, W. Q. J. Org. Chem. 1988, 53, 5685-5689.

${ }^{2}$ Arduengo, A. J., III; Gentry, F. P., Jr.; Taverkere, P. K.; Simmons, H. E., III U.S. Patent 6,177,575, 2001; Chem. Abst. 2001, 134, 115958.
} 


\section{Synthesis of 2-Alkylimidazoles}

Method A. Treatment of imidazole with formaldehyde and dimethylamine hydrochloride gave 1( $N, N^{\prime}$-dimethylaminomethyl)imidazole as described. ${ }^{1}$ Lithiation $(\mathrm{BuLi} /$ hexanes) of that protected derivative in THF, alkylation, and acid-catalyzed hydrolysis of the protecting group gave the $\mathrm{C} 2$ alkylated product.

2-Dodecylimidazole. A solution of 1 -( $N, N^{\prime}$-dimethylaminomethyl)imidazole ${ }^{1}(1.32 \mathrm{~g}$, $10.6 \mathrm{mmol})$ in dried THF $(40 \mathrm{~mL})$ was cooled to $-78{ }^{\circ} \mathrm{C}$ under $\mathrm{N}_{2}$, and $\mathrm{BuLi} /$ hexanes $(1.6 \mathrm{M}, 8.0$ $\mathrm{mL}, 12.8 \mathrm{mmol}$ ) was added dropwise. The solution was stirred for $1.5 \mathrm{~h}$ at $-78{ }^{\circ} \mathrm{C}$, and a solution of 1-iodododecane $(4.0 \mathrm{~mL}, 4.8 \mathrm{~g}, 16.2 \mathrm{mmol})$ in THF $(5 \mathrm{~mL})$ was added. Stirring at $-78{ }^{\circ} \mathrm{C}$ was continued for $30 \mathrm{~h}$, and the reaction mixture was allowed to warm to ambient temperature. $\mathrm{HCl} / \mathrm{H}_{2} \mathrm{O}(37 \% \mathrm{w} / \mathrm{w}, 2 \times 6 \mathrm{~mL})$ was added, and the solution was stirred overnight at ambient temperature and neutralized $\left(\mathrm{NaHCO}_{3} / \mathrm{H}_{2} \mathrm{O}\right)$. Volatiles were evaporated in vacuo, and the residue was extracted with $\mathrm{CHCl}_{3}(3 \times 100 \mathrm{~mL})$. The combined organic phase was dried $\left(\mathrm{Na}_{2} \mathrm{SO}_{4}\right)$, and volatiles were evaporated in vacuo. The residue was chromatographed (EtOAc) to give a solid (1.96 g, 79\%) that was recrystallized $\left(\mathrm{CH}_{2} \mathrm{Cl}_{2}\right)$ to give the title compound $(1.51 \mathrm{~g}, 59 \%): \mathrm{mp}$

76.5-77.5 ${ }^{\circ} \mathrm{C} ;{ }^{1} \mathrm{H}$ NMR $\left(300 \mathrm{MHz}, \mathrm{CDCl}_{3}\right) \delta 9.48(\mathrm{~s}, 1 \mathrm{H}), 6.97(\mathrm{~s}, 2 \mathrm{H}), 2.76(\mathrm{t}, J=7.5 \mathrm{~Hz}, 2 \mathrm{H})$, 1.75 (quint, $J=7.5 \mathrm{~Hz}, 2 \mathrm{H}), 1.33-1.27(\mathrm{~m}, 18 \mathrm{H}), 0.90(\mathrm{t}, J=6.8 \mathrm{~Hz}, 3 \mathrm{H}) ;{ }^{13} \mathrm{C}$ NMR $(75 \mathrm{MHz}$, $\left.\mathrm{CDCl}_{3}\right) \delta 149.2,121.9,32.2,29.94,29.90,29.8,29.6,28.92,28.90,23.0,14.4 ; \mathrm{HRMS} \mathrm{m} / \mathrm{z}$ 236.2240 $\left(\mathrm{M}^{+}\left[\mathrm{C}_{15} \mathrm{H}_{28} \mathrm{~N}_{2}\right]=236.2252\right)$. Anal. Calcd for $\mathrm{C}_{15} \mathrm{H}_{28} \mathrm{~N}_{2}: \mathrm{C}, 76.21 ; \mathrm{H}, 11.94 ; \mathrm{N}, 11.85$. Found: C, 76.42; H, 12.00; N, 12.02 .

Early chromatographic fractions also contained 1,2-bis(dodecyl)imidazole: ${ }^{13} \mathrm{C}$ NMR (75 $\left.\mathrm{MHz}, \mathrm{CDCl}_{3}\right) \delta 147.4,120.7,119.2,48.0,32.2,30.5,29.9,29.8,29.73,29.70,29.6,29.40$ 29.36, 29.28, 29.27, 28.3, 26.7, 25.2, 22.9, 14.4; MS m/z $404\left(\mathrm{M}^{+}\left[\mathrm{C}_{27} \mathrm{H}_{52} \mathrm{~N}_{2}\right]=404\right)$. 
Method B. A mixture of an aliphatic aldehyde, ammonium bicarbonate, and glyoxal in water was stirred overnight at ambient temperature as described. ${ }^{2}$ Volatiles were evaporated to give the residual 2-alkylimidazoles.

2-Propylimidazole. Butanal $(9.2 \mathrm{~mL}, 7.52 \mathrm{~g}, 104 \mathrm{mmol})$ was added to a suspension of $\mathrm{NH}_{4} \mathrm{HCO}_{3}(16.45 \mathrm{~g}, 208.1 \mathrm{mmol})$ in $\mathrm{H}_{2} \mathrm{O}(10 \mathrm{~mL})$ and glyoxal $/ \mathrm{H}_{2} \mathrm{O}(40 \% \mathrm{w} / \mathrm{w}, 11.9 \mathrm{~mL}, 15.09$ g, $104.0 \mathrm{mmol})$. The mixture was stirred at ambient temperature overnight, and volatiles were evaporated. The residue was extracted with THF, and volatiles were evaporated to give the crude product ( 11 g). Chromatography $\left[\mathrm{CH}_{2} \mathrm{Cl}_{2} \rightarrow \mathrm{MeOH} / \mathrm{CH}_{2} \mathrm{Cl}_{2}(1: 60 \rightarrow 1: 30)\right]$ removed the byproduct imidazole (formed by cyclization with formaldehyde, which is a contaminant in the glyoxal solution) to give 2-propylimidazole (7.45 g, 65\%): ${ }^{1} \mathrm{H} \mathrm{NMR}\left(500 \mathrm{MHz}, \mathrm{CDCl}_{3}\right) \delta 11.50$ (s, 1H), $6.96(\mathrm{~s}, 2 \mathrm{H}), 2.72(\mathrm{t}, J=7.4 \mathrm{~Hz}, 2 \mathrm{H}), 1.77$ (sext, $J=7.4 \mathrm{~Hz}, 2 \mathrm{H}), 0.98(\mathrm{t}, J=7.4 \mathrm{~Hz}$, $3 \mathrm{H}) ;{ }^{13} \mathrm{C} \mathrm{NMR}\left(125 \mathrm{MHz}, \mathrm{CDCl}_{3}\right) \delta 149.1,121.4,30.7,22.3,14.0$.

\section{Synthesis of 6-(Imidazol-1-yl)purine Derivatives}

6-(2-Propylimidazol-1-yl)purine (3b). A stirred suspension of 9 (1.58 g, $4.0 \mathrm{mmol})$, $\mathrm{Ph}_{3} \mathrm{P}(2.58 \mathrm{~g}, 9.6 \mathrm{mmol}), \mathrm{I}_{2}(2.14 \mathrm{~g}, 8.32 \mathrm{mmol})$, and 2-propylimidazole $(1.60 \mathrm{~g}, 14.4 \mathrm{mmol})$ in $\mathrm{EtN}(\mathrm{iPr})_{2}(3.6 \mathrm{~mL}, 2.67 \mathrm{~g}, 20.2 \mathrm{mmol})$ and dried toluene $(40 \mathrm{~mL})$ was stirred for $4 \mathrm{~h}$ at $95{ }^{\circ} \mathrm{C}$. Volatiles were evaporated in vacuo, and the residue was extracted with boiling EtOAc. The combined EtOAc extracts were evaporated to dryness, and the residue was chromatographed $\left(\mathrm{CH}_{2} \mathrm{Cl}_{2} / \mathrm{MeOH}, 1: 40\right)$ to give a solid contaminated with $\mathrm{Ph}_{3} \mathrm{PO}$. This material was dissolved in $\mathrm{AcOH}(160 \mathrm{~mL})$, and $\mathrm{AcCl}(2.2 \mathrm{~mL}, 2.43 \mathrm{~g}, 31 \mathrm{mmol})$ was added. The solution was stirred overnight at $65{ }^{\circ} \mathrm{C}$, and volatiles were evaporated in vacuo. The residue was dissolved in $\mathrm{CH}_{2} \mathrm{Cl}_{2}$ and extracted with $0.1 \mathrm{~N} \mathrm{NaOH} / \mathrm{H}_{2} \mathrm{O}$. The aqueous layer was washed $\left(\mathrm{CH}_{2} \mathrm{Cl}_{2}\right)$, and $\mathrm{CO}_{2}$ was bubbled into the solution. The resulting precipitate was filtered and washed thoroughly $\left(\mathrm{H}_{2} \mathrm{O}\right)$ to 
give a solid (0.66 g, 72\%). This material was dissolved $(\mathrm{MeOH})$ and decolorized (charcoal). Recrystallization $(\mathrm{MeOH})$ gave $\mathbf{3 b}$ as a colorless solid: $\mathrm{mp} 242.5-243.5{ }^{\circ} \mathrm{C}$; $\mathrm{UV}(\mathrm{MeOH}) \max$ $278 \mathrm{~nm}(\varepsilon 13700)$, $\min 235 \mathrm{~nm}\left(\varepsilon \text { 5000); }{ }^{1} \mathrm{H} \text { NMR (300 MHz, DMSO-d }\right)_{6} \delta 13.90$ (br s, $\left.1 \mathrm{H}\right)$, 8.86, 8.69, $8.36(3 \times \mathrm{s}, 3 \times 1 \mathrm{H}), 7.07(\mathrm{~d}, J=1.5 \mathrm{~Hz}, 1 \mathrm{H}), 3.18(\mathrm{t}, J=7.3 \mathrm{~Hz}, 2 \mathrm{H}), 1.72(\mathrm{sext}, J=$ $7.3 \mathrm{~Hz}, 2 \mathrm{H}), 0.93(\mathrm{t}, J=7.3 \mathrm{~Hz}, 3 \mathrm{H}) ;{ }^{13} \mathrm{C} \mathrm{NMR}\left(75 \mathrm{MHz}, \mathrm{CDCl}_{3}\right) \delta 155.5,152.0,149.8,146.9$, $145.8,128.5,123.6,121.4,32.2,21.5,14.5 ;$ HRMS $m / z 228.1109\left(\mathrm{M}^{+}\left[\mathrm{C}_{11} \mathrm{H}_{12} \mathrm{~N}_{6}\right]=228.1123\right)$. Anal. Calcd for $\mathrm{C}_{11} \mathrm{H}_{12} \mathrm{~N}_{6}$ : C, 57.88; H, 5.30; N, 36.82. Found: C, 58.09; H, 5.19; N, 37.00.

6-(2-Hexylimidazol-1-yl)purine (3c). A stirred suspension of 9 (3.93 g, $10 \mathrm{mmol}), \mathrm{Ph}_{3} \mathrm{P}$ (6.41 g, $21 \mathrm{mmol}), \mathrm{I}_{2}(5.36 \mathrm{~g}, 20.7 \mathrm{mmol})$, and crude 2-hexylimidazole $(6.48 \mathrm{~g}, 42.7 \mathrm{mmol})$ in $\operatorname{EtN}(i P r))_{2}(9.9 \mathrm{~mL}, 7.36 \mathrm{~g}, 56.7 \mathrm{mmol})$ and dried toluene $(100 \mathrm{~mL})$ was heated for $12 \mathrm{~h}$ at $95{ }^{\circ} \mathrm{C}$. Volatiles were evaporated in vacuo, and the residue was extracted with boiling EtOAc. The combined EtOAc extracts were evaporated to dryness, and the residue was chromatographed $\left(\mathrm{MeOH} / \mathrm{CH}_{2} \mathrm{Cl}_{2}, 1: 40\right)$. The derived solid was dissolved in $\mathrm{AcOH}(387 \mathrm{~mL})$, and $\mathrm{AcCl}(4.2 \mathrm{~mL}$, $4.64 \mathrm{~g}, 59 \mathrm{mmol}$ ) was added. The solution was stirred for 2 days at $65{ }^{\circ} \mathrm{C}$, and volatiles were evaporated in vacuo. The residue was dissolved in $\mathrm{CHCl}_{3}$, and the solution was washed $\left(\mathrm{NaHCO}_{3} / \mathrm{H}_{2} \mathrm{O}\right)$ and dried $\left(\mathrm{Na}_{2} \mathrm{SO}_{4}\right)$. Volatiles were evaporated in vacuo, and the residue was chromatographed $\left(\mathrm{MeOH} / \mathrm{CH}_{2} \mathrm{Cl}_{2}, 1: 20\right)$ and recrystallized $(\mathrm{MeOH})$ to give $3 \mathrm{c}(1.1 \mathrm{~g}, 41 \%)$ : $\mathrm{mp}$ 224.5-225 ${ }^{\circ} \mathrm{C}$; UV (MeOH) $\max 278 \mathrm{~nm}(\varepsilon 12200)$, $\min 232 \mathrm{~nm}(\varepsilon 2200) ;{ }^{1} \mathrm{H}$ NMR (500 MHz, DMSO-d $\left.d_{6}\right) \delta 13.89,8.84,8.68,8.33(4 \times \mathrm{s}, 4 \times 1 \mathrm{H}), 7.04(\mathrm{~d}, J=1.5 \mathrm{~Hz}, 1 \mathrm{H}), 3.18(\mathrm{t}, J=7.4 \mathrm{~Hz}$ 2H), 1.66 (quint, $J=7.6 \mathrm{~Hz}, 2 \mathrm{H}$ ), 1.32 (quint, $J=7.1 \mathrm{~Hz}, 2 \mathrm{H}), 1.20-1.24(\mathrm{~m}, 4 \mathrm{H}), 0.82$ (t, $J=$ $7.1 \mathrm{~Hz}, 3 \mathrm{H}) ;{ }^{13} \mathrm{C}$ NMR ( $\left.125 \mathrm{MHz}, \mathrm{DMSO}-d_{6}\right) \delta 154.8,151.2,149.3,146.1,145.0,127.8,122.8$, 120.6, 30.9, 29.4, 28.3, 27.3, 22.0, 13.8; HRMS (EI) $m / z 270.1580\left(\mathrm{M}^{+}\left[\mathrm{C}_{14} \mathrm{H}_{18} \mathrm{~N}_{6}\right]=270.1593\right)$. Anal. Calcd for $\mathrm{C}_{14} \mathrm{H}_{18} \mathrm{~N}_{6}$ : C, 62.20; H, 6.71; N, 31.09. Found: C, 62.32; H, 6.86; N, 31.25. 
6-(2-Dodecylimidazol-1-yl)purine (3d). A stirred suspension of 9 (100 mg, $0.25 \mathrm{mmol})$, $\mathrm{Ph}_{3} \mathrm{P}(160 \mathrm{mg}, 0.6 \mathrm{mmol}), \mathrm{I}_{2}(140 \mathrm{mg}, 0.52 \mathrm{mmol})$, and 2-dodecylimidazole (210 mg, $\left.0.9 \mathrm{mmol}\right)$ in $\operatorname{EtN}(\mathrm{iPr})_{2}(0.22 \mathrm{~mL}, 160 \mathrm{mg}, 1.26 \mathrm{mmol})$ and dried toluene $(2.5 \mathrm{~mL})$ was heated for $12 \mathrm{~h}$ at 95 ${ }^{\circ} \mathrm{C}$. Volatiles were evaporated in vacuo, and the residue was extracted with boiling EtOAc. The combined EtOAc extracts were evaporated to dryness, and the residue was chromatographed (EtOAc/hexanes, 1:1 $\rightarrow$ EtOAc). The derived solid was dissolved in $\mathrm{AcOH}(10 \mathrm{~mL})$, and $\mathrm{AcCl}$ $(0.11 \mathrm{~mL}, 103 \mathrm{mg}, 1.5 \mathrm{mmol})$ was added. The solution was stirred overnight at $65{ }^{\circ} \mathrm{C}$ and then evaporated to dryness. The residue was dissolved in $\mathrm{CHCl}_{3}$, and the solution was washed [saturated $\mathrm{NaHCO}_{3} / \mathrm{H}_{2} \mathrm{O}(3 \times 50 \mathrm{~mL})$ ] and dried $\left(\mathrm{Na}_{2} \mathrm{SO}_{4}\right)$. Volatiles were evaporated in vacuo, and the residue was chromatographed $\left(\mathrm{MeOH} / \mathrm{CH}_{2} \mathrm{Cl}_{2}, 1: 12\right)$ to give a solid that was contaminated with 2-dodecylimidazole. Recrystallization (MeOH) gave 3d (48 mg, 54\%): mp 210-210.5 ${ }^{\circ} \mathrm{C}$; UV (MeOH) $\max 278 \mathrm{~nm}(\varepsilon 14000)$, $\min 242 \mathrm{~nm}(\varepsilon 6000) ;{ }^{1} \mathrm{H}$ NMR $(300 \mathrm{MHz}$, DMSO-d $\left.d_{6}\right) \delta 13.87,8.83,8.67,8.33,7.04(5 \times \mathrm{s}, 5 \times 1 \mathrm{H}), 3.18(\mathrm{t}, J=7.3 \mathrm{~Hz}, 2 \mathrm{H}), 1.66$ (quint, $J$ $=7.3 \mathrm{~Hz}, 2 \mathrm{H}), 1.29-1.20(\mathrm{~m}, 18 \mathrm{H}), 0.84(\mathrm{t}, J=7.0 \mathrm{~Hz}, 3 \mathrm{H}) ;{ }^{13} \mathrm{C} \mathrm{NMR}\left(125 \mathrm{MHz}, \mathrm{CDCl}_{3}\right) \delta$ $154.9,151.2,149.2,146.1,145.0,127.8,122.9,120.6,31.3,29.3,28.99,28.96,28.86,28.7,27.4$, 22.1, 13.9; HRMS m/z $354.2525\left(\mathrm{M}^{+}\left[\mathrm{C}_{20} \mathrm{H}_{30} \mathrm{~N}_{6}\right]=354.2532\right)$. Anal. Calcd for $\mathrm{C}_{20} \mathrm{H}_{30} \mathrm{~N}_{6}$ : $\mathrm{C}$, 67.76; H, 8.53; N, 23.71. Found: C, 67.90; H, 8.66; N, 23.86.

2-Chloro-6-(2-propylimidazol-1-yl)purine (8b). Treatment of 12 (1.71 g, $3.83 \mathrm{mmol})$ with 2-propylimidazole in $\mathrm{CH}_{3} \mathrm{CN}$ and chromatography (as described for 13a) gave 9-(2,3,5-tri$O$-acetyl- $\beta$-D-ribofuranosyl)-2-chloro-6-(2-propylimidazol-1-yl)purine (13b). AcCl $(4.0 \mathrm{~mL}$, $4.42 \mathrm{~g}, 56.3 \mathrm{mmol})$ was added to a solution of $\mathbf{1 3 b}(4.99 \mathrm{~g}, 9.6 \mathrm{mmole})$ in HOAc $(400 \mathrm{~mL})$, the mixture was stirred for $1.5 \mathrm{~h}$ at $65{ }^{\circ} \mathrm{C}$ in a sealed flask (reaction essentially complete, TLC), and volatiles were evaporated in vacuo. The residue was washed $\left(\mathrm{CH}_{2} \mathrm{Cl}_{2}\right)$ and then dissolved in 
$\mathrm{NaOH} / \mathrm{H}_{2} \mathrm{O}(0.1 \mathrm{M}) . \mathrm{CO}_{2}$ was bubbled into the solution, and the resulting precipitate $(2.20 \mathrm{~g}$, $88 \%$ ) was filtered. Recrystallization of this material from $\mathrm{MeOH}$ gave $8 \mathbf{b}(1.93 \mathrm{~g}, 77 \%): \mathrm{mp}$ 224.5-225 ${ }^{\circ} \mathrm{C}$; UV (MeOH) $\max$ 215, $288 \mathrm{~nm}(\varepsilon 25800,16700)$, min $241 \mathrm{~nm}(\varepsilon 4500) ;{ }^{1} \mathrm{H}$ NMR (500 MHz, DMSO-d $\left.{ }_{6}\right) \delta 14.04($ br s, $1 \mathrm{H}), 8.69,8.43,7.06(3 \times \mathrm{s}, 3 \times 1 \mathrm{H}), 3.12(\mathrm{t}, J=7.5$ $\mathrm{Hz}, 2 \mathrm{H}), 1.72$ (sext, $J=7.3 \mathrm{~Hz}, 2 \mathrm{H}), 0.95(\mathrm{t}, J=7.3 \mathrm{~Hz}, 3 \mathrm{H}) ;{ }^{13} \mathrm{C}$ NMR ( $\left.125 \mathrm{MHz}, \mathrm{DMSO}-d_{6}\right) \delta$ $157.3,151.7,150.2,147.2,146.6,128.9,122.5,121.1,32.4,21.5,14.5 ;$ HRMS m/z 262.0723 $\left(\mathrm{M}^{+}\left[\mathrm{C}_{11} \mathrm{H}_{11} \mathrm{ClN}_{6}\right]=262.0734\right)$. Anal. Calcd for $\mathrm{C}_{11} \mathrm{H}_{11} \mathrm{ClN}_{6}: \mathrm{C}, 50.29 ; \mathrm{H}, 4.22 ; \mathrm{N}, 31.99$. Found: C, 50.02; H, 4.28; N, 31.64.

2-Chloro-6-(2-butylimidazol-1-yl)purine (8c). AcCl (1.67 mL, 1.84 g, 23.5 mmol) was added to crude 9-(2,3,5-tri-O-acetyl- $\beta$-D-ribofuranosyl)-6-(2-butylimidazol-1-yl)-2-chloropurine (13c) $(2.16 \mathrm{~g}, 4 \mathrm{mmol})$ in acetic acid $(167 \mathrm{~mL})$, the mixture was stirred for $23 \mathrm{~h}$ at $65{ }^{\circ} \mathrm{C}$ in a sealed flask (reaction complete, TLC), and volatiles were evaporated in vacuo. The residue was washed $\left(\mathrm{CH}_{2} \mathrm{Cl}_{2}\right)$ and then dissolved in $\mathrm{NaOH} / \mathrm{H}_{2} \mathrm{O}(0.1 \mathrm{M}, 130 \mathrm{~mL})$. Precipitation (bubbling in $\left.\mathrm{CO}_{2}\right)$ gave a solid $(0.76 \mathrm{~g}, 57 \%)$ that was recrystallized $(\mathrm{MeOH})$ to give $8 \mathrm{c}(0.58 \mathrm{~g}, 44 \%): \mathrm{mp}$ 247-247.5 ${ }^{\circ} \mathrm{C}$; UV (MeOH) $\max 214,254,288 \mathrm{~nm}(\varepsilon 25600,4700,13900), \min 239,257 \mathrm{~nm}(\varepsilon$ 3800, 4600); ${ }^{1} \mathrm{H}$ NMR (500 MHz, DMSO-d $) \delta 14.05,8.71,8.44(3 \times \mathrm{s}, 3 \times 1 \mathrm{H}), 7.07(\mathrm{~d}, J=1.5$ $\mathrm{Hz}, 1 \mathrm{H}), 3.17$ (t, $J=7.7 \mathrm{~Hz}, 2 \mathrm{H}), 1.70$ (quint, $J=7.6 \mathrm{~Hz}, 2 \mathrm{H}$ ), 1.39 (sext, $J=7.6 \mathrm{~Hz}, 2 \mathrm{H}$ ), 0.91 $(\mathrm{t}, J=7.4 \mathrm{~Hz}, 3 \mathrm{H}) ;{ }^{13} \mathrm{C} \mathrm{NMR}\left(125 \mathrm{MHz}, \mathrm{DMSO}-d_{6}\right) \delta 156.3,151.0,149.5,146.4,145.7,128.1$, 121.7, 120.3, 29.5, 29.3, 21.9, 13.6; HRMS m/z 277.0973 $\left(\mathrm{MNa}^{+}\left[\mathrm{C}_{12} \mathrm{H}_{14} \mathrm{ClN}_{6} \mathrm{Na}\right]=277.0968\right)$. Anal. Calcd for $\mathrm{C}_{12} \mathrm{H}_{14} \mathrm{ClN}_{6}$ : C, 52.08; H, 4.74; N, 30.37. Found: C, 51.96; H, 4.85; N, 30.52 .

2-Chloro-6-(2-pentylimidazol-1-yl)purine (8d). AcCl (1.4 mL, 1.55 g, 19.7 mmol) was added to a solution of crude 9-(2,3,5-tri-O-acetyl- $\beta$-D-ribofuranosyl)-2-chloro-6-(2- 
pentylimidazol-1-yl)purine (13d) $(1.82 \mathrm{~g}, 3.3 \mathrm{mmol})$ in acetic acid $(136 \mathrm{~mL})$, the mixture was stirred for $23 \mathrm{~h}$ at $65{ }^{\circ} \mathrm{C}$ in a sealed flask (reaction complete, TLC), and volatiles were evaporated in vacuo. The residue was washed $\left(\mathrm{CH}_{2} \mathrm{Cl}_{2}\right)$ and then dissolved in $\mathrm{NaOH} / \mathrm{H}_{2} \mathrm{O}(0.1 \mathrm{M}, 60 \mathrm{~mL})$. Precipitation (bubbling in $\left.\mathrm{CO}_{2}\right)$ gave a solid $(0.34 \mathrm{~g}, 48 \%)$ that was recrystallized $(\mathrm{MeOH})$ to give 8d (0.27 g, 38\%): mp 254.5-255 ${ }^{\circ} \mathrm{C}$; UV (MeOH) max 214, 254, $288 \mathrm{~nm}(\varepsilon 27$ 200, 13 500, 4600), $\min 240,258 \mathrm{~nm}(\varepsilon 3800,4600) ;{ }^{1} \mathrm{H}$ NMR (500 MHz, DMSO-d $\left.d_{6}\right) \delta 14.05,8.70,8.44(3 \times$ s, $3 \times 1 \mathrm{H}), 7.07(\mathrm{~d}, J=1.5 \mathrm{~Hz}, 1 \mathrm{H}), 3.15(\mathrm{t}, J=7.6 \mathrm{~Hz}, 2 \mathrm{H}), 1.71$ (quint, $J=7.5 \mathrm{~Hz}, 2 \mathrm{H}$ ), $1.29-$ $1.38(\mathrm{~m}, 4 \mathrm{H}), 0.86(\mathrm{t}, J=7.2 \mathrm{~Hz}, 3 \mathrm{H}) ;{ }^{13} \mathrm{C}$ NMR $\left(125 \mathrm{MHz}\right.$, DMSO-d $\left.d_{6}\right) \delta 156.2,151.0,149.5$, $146.4,145.7,128.1,121.7,120.3,31.0,29.6,27.1,21.8,13.8 ;$ HRMS $\mathrm{m} / \mathrm{z} 291.1138\left(\mathrm{MNa}^{+}\right.$ $\left.\left[\mathrm{C}_{13} \mathrm{H}_{16} \mathrm{ClN}_{6}\right]=291.1125\right)$. Anal. Calcd for $\mathrm{C}_{13} \mathrm{H}_{15} \mathrm{ClN}_{6}: \mathrm{C}, 53.70 ; \mathrm{H}, 5.22 ; \mathrm{N}, 28.90$. Found: $\mathrm{C}$, 53.55; H, 5.22; N, 29.00.

2-Chloro-6-(4,5-diphenyllimidazol-1-yl)purine (8e). $\mathrm{AcCl}(0.68 \mathrm{~mL}, 0.75 \mathrm{~g}, 9.6 \mathrm{mmol})$ was added to a solution of 9-(2,3,5-tri-O-acetyl- $\beta$-D-ribofuranosyl)-2-chloro-6-(4,5-diphenylimidazol-1-yl)purine (13e) $(1.41 \mathrm{~g}, 1.7 \mathrm{mmol})$ in acetic acid $(69 \mathrm{~mL})$, the mixture was stirred for $60 \mathrm{~h}$ at $65^{\circ} \mathrm{C}$ in a sealed flask (reaction complete, TLC), and volatiles were evaporated in vacuo. The residue was washed $\left(\mathrm{CH}_{2} \mathrm{Cl}_{2}\right)$ and then dissolved in $\mathrm{NaOH} / \mathrm{H}_{2} \mathrm{O}(0.1 \mathrm{M})$. Precipitation (bubbling in $\left.\mathrm{CO}_{2}\right)$ gave a solid $(0.41 \mathrm{~g}, 67 \%)$ that was recrystallized $(\mathrm{MeOH})$ to give 8e: $\mathrm{mp}$ 277.5-278 ${ }^{\circ} \mathrm{C}$; UV (MeOH) $\max 277 \mathrm{~nm}(\varepsilon 16100)$, min $264 \mathrm{~nm}(\varepsilon 14800) ;{ }^{1} \mathrm{H}$ NMR (500 MHz, DMSO-d $\left.d_{6}\right) \delta 14.04,8.84,8.73(3 \times \mathrm{s}, 3 \times 1 \mathrm{H}), 7.20-7.49(\mathrm{~m}, 10 \mathrm{H}) ;{ }^{13} \mathrm{C} \mathrm{NMR}(125 \mathrm{MHz}$, DMSO- $\left.d_{6}\right) \delta 157.2,151.6,147.4,146.2,139.7,139.5,134.3,131.3,131.1,129.02,128.98$ 128.92, 128.08, 127.8, 127.6, 124.0; HRMS m/z $395.0792\left(\mathrm{MNa}^{+}\left[\mathrm{C}_{20} \mathrm{H}_{13} \mathrm{ClN}_{6} \mathrm{Na}\right]=395.0788\right)$. Anal. Calcd for $\mathrm{C}_{20} \mathrm{H}_{13} \mathrm{ClN}_{6}$ : C, 64.43; H, 3.51; N, 22.54. Found: C, 64.29; H, 3.78; N, 22.53 . 
X-Ray crystal structure of 6-(1,2,4-triazol-4-yl)purine•pyridine (Compound B, Fig. 1).

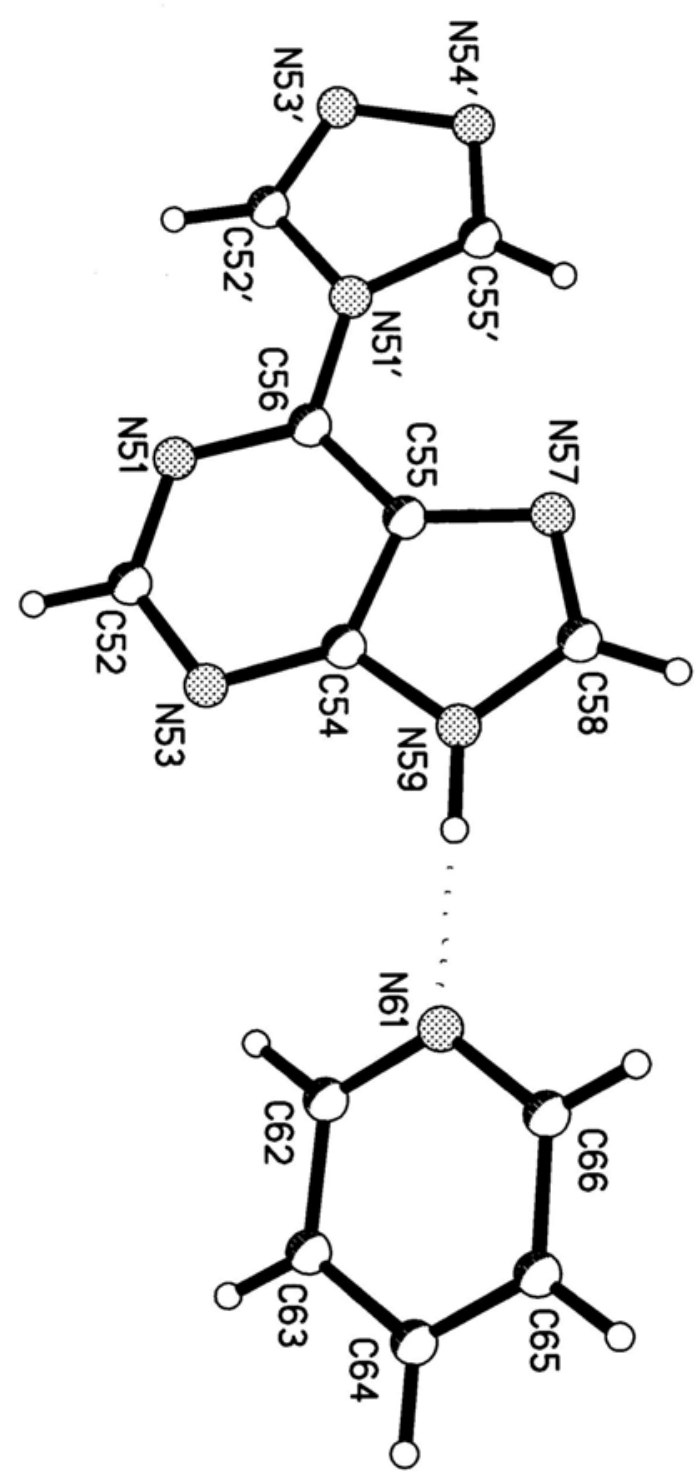


X-Ray crystal structure of 6-(2-butylimidazol-1-yl)-2-chloropurine (Compound 8c, Scheme 2).

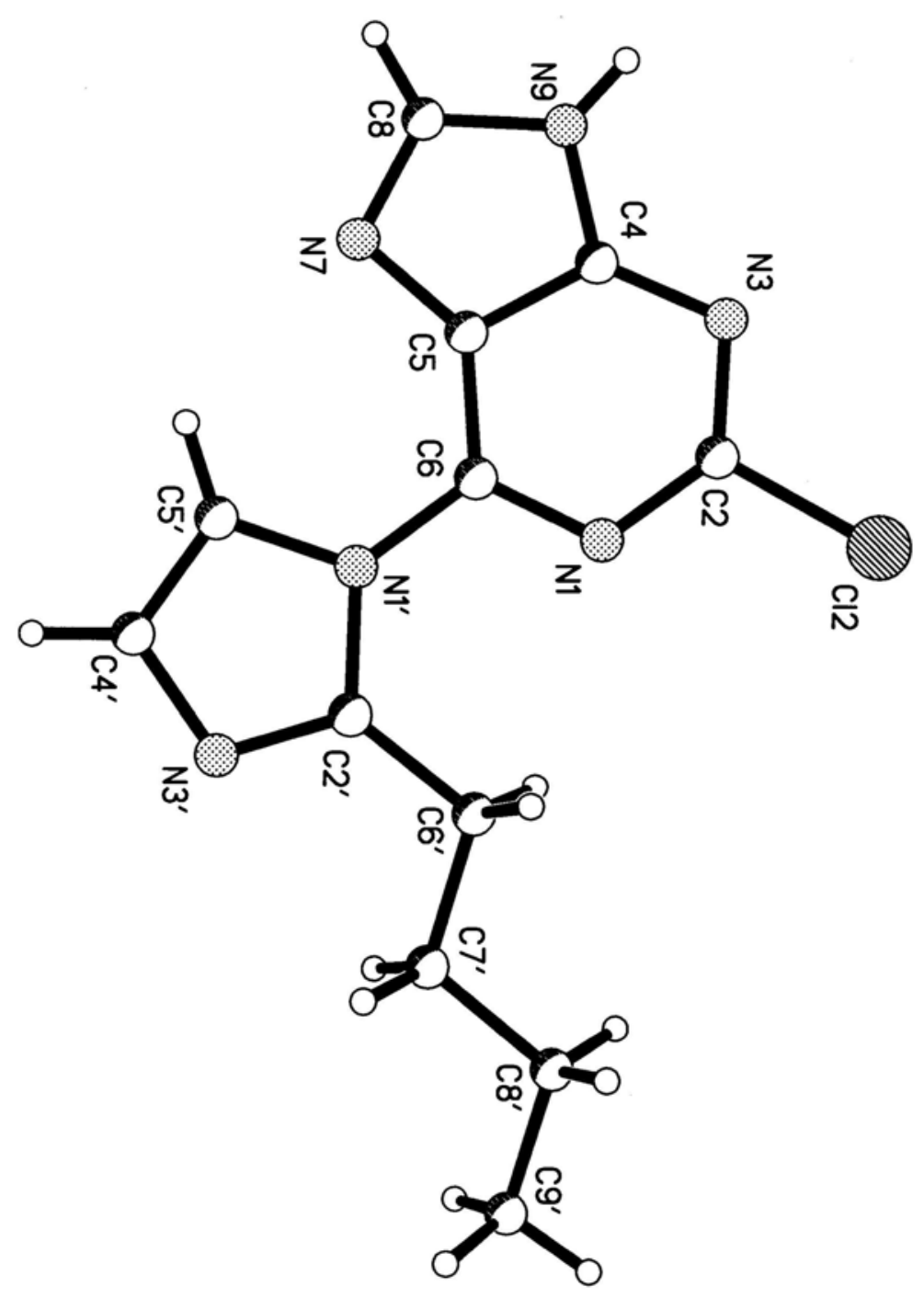


X-Ray crystal structure of 9-(5-O-acetyl- $\beta$-D-ribofuranosyl)-6-(imidazol-1-yl)purine (Compound D, Fig. 2).

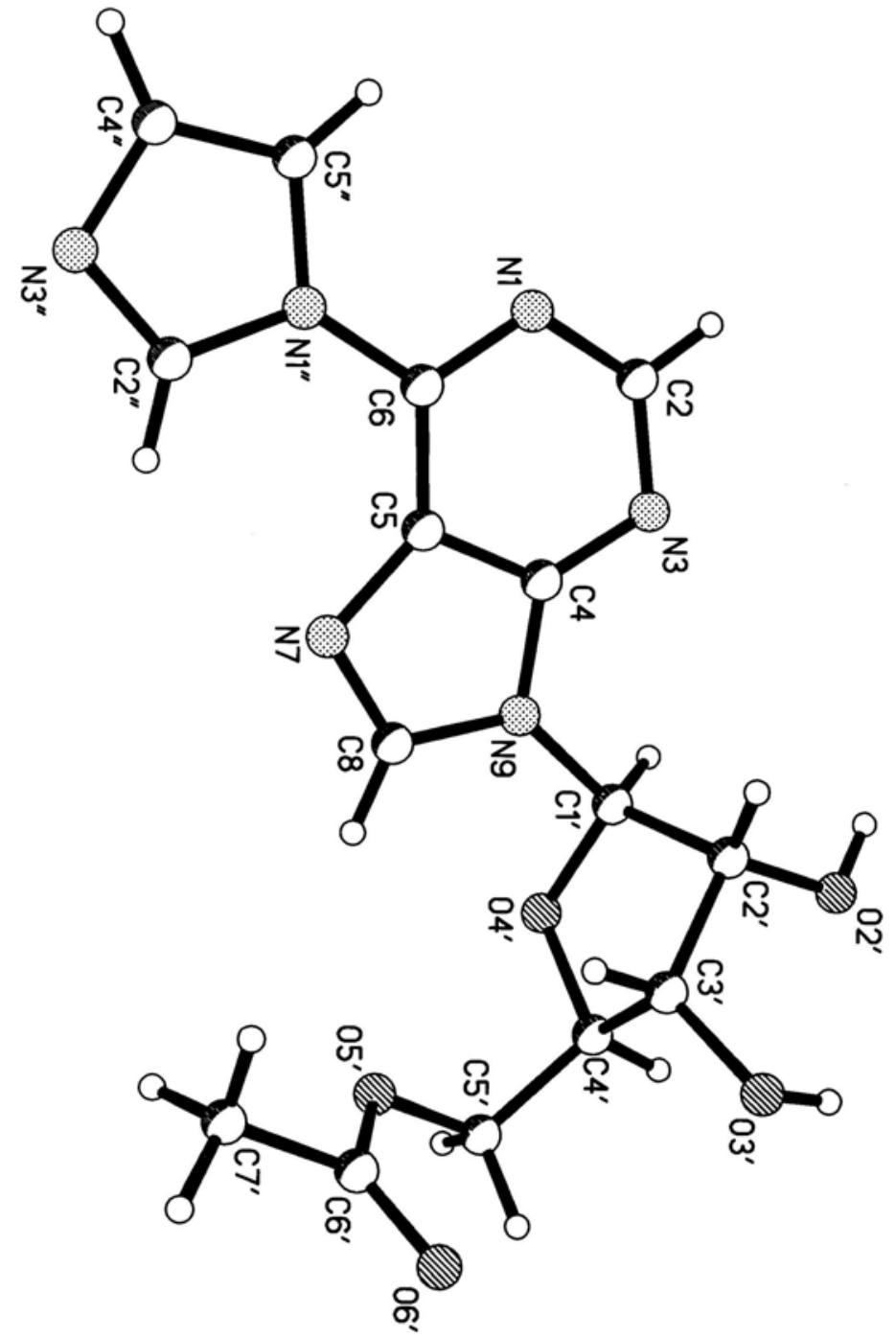


X-Ray crystal structure of 9-(2,3,5-tri-O-acetyl- $\beta$-D-ribofuranosyl)-2-chloro-6-(4,5-

diphenylimidazol-1-yl)purine (Compound E, Fig. 2).

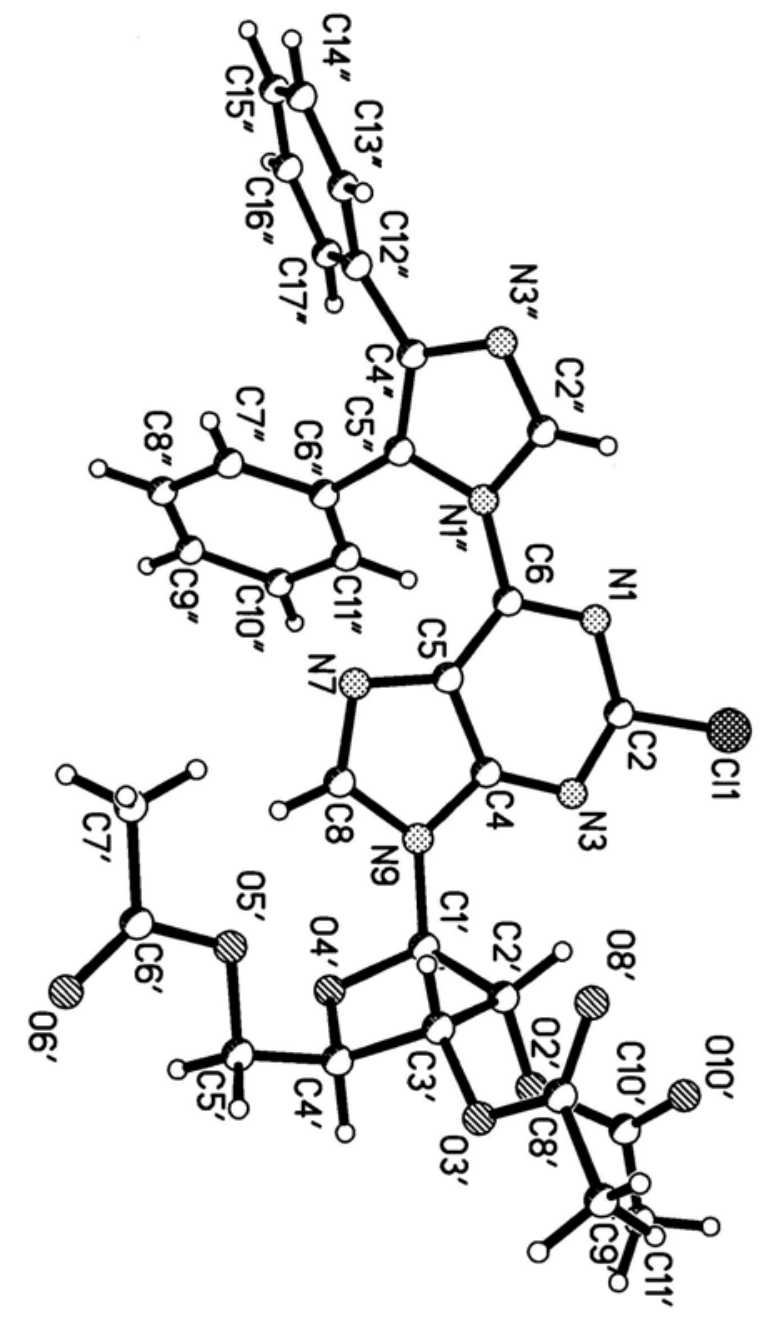


X-Ray crystal structure of 9-(5-O-tert-butyldimethylsilyl-2,3-dideoxy-3-iodo- $\beta$-D-threopentofuranosyl)-6-(2,5-dimethylpyrrol-1-yl)purine (Compound F, Fig. 2).

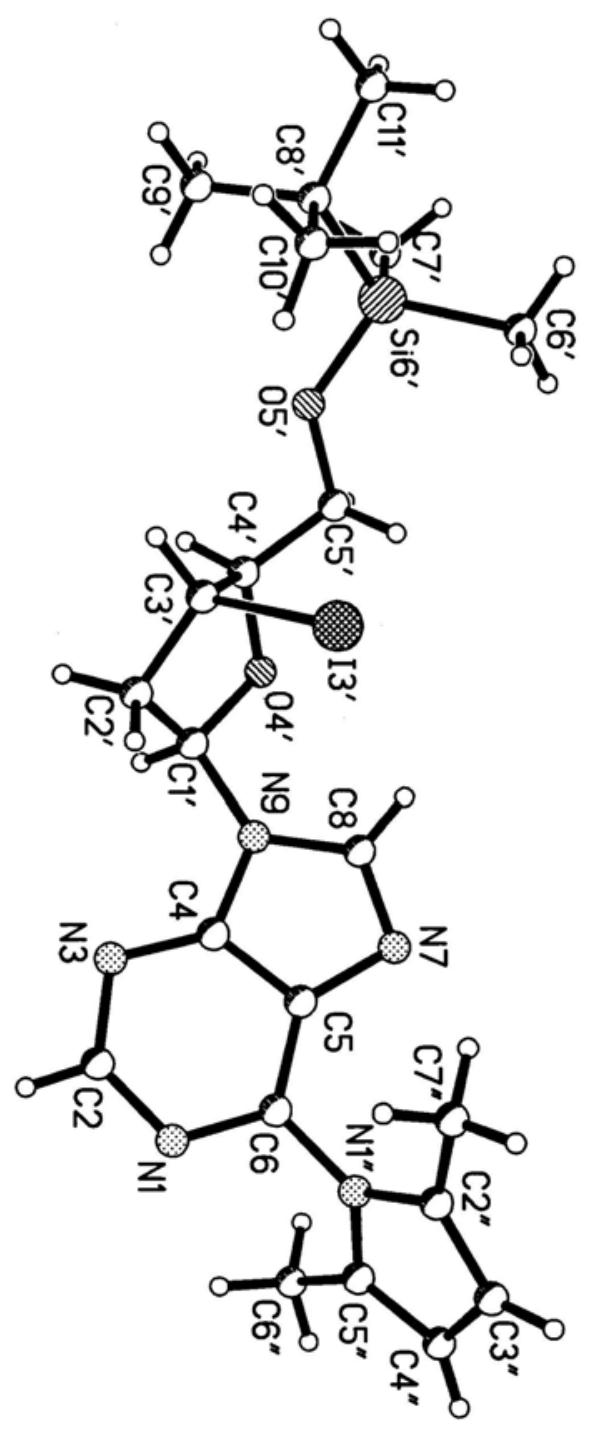




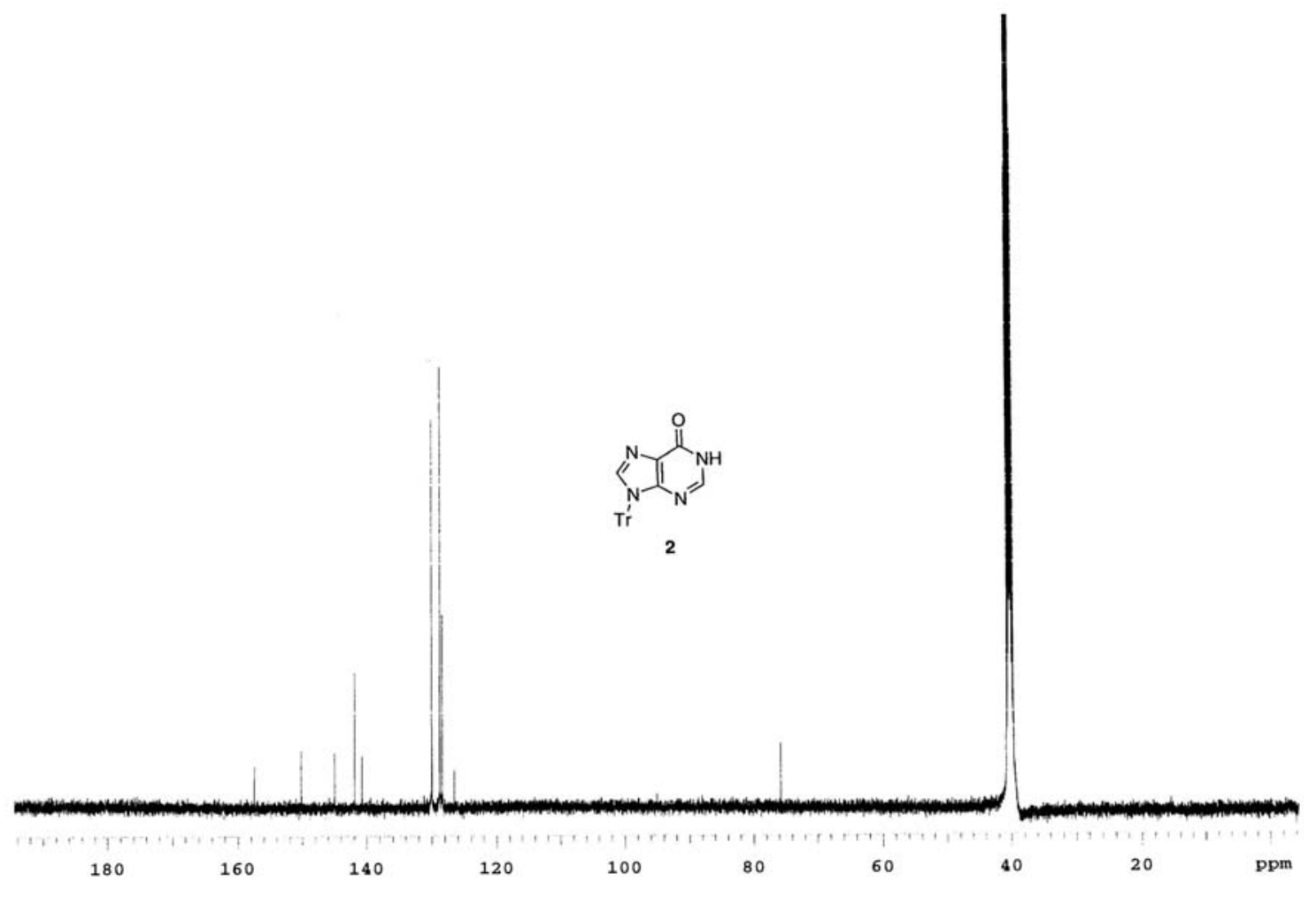




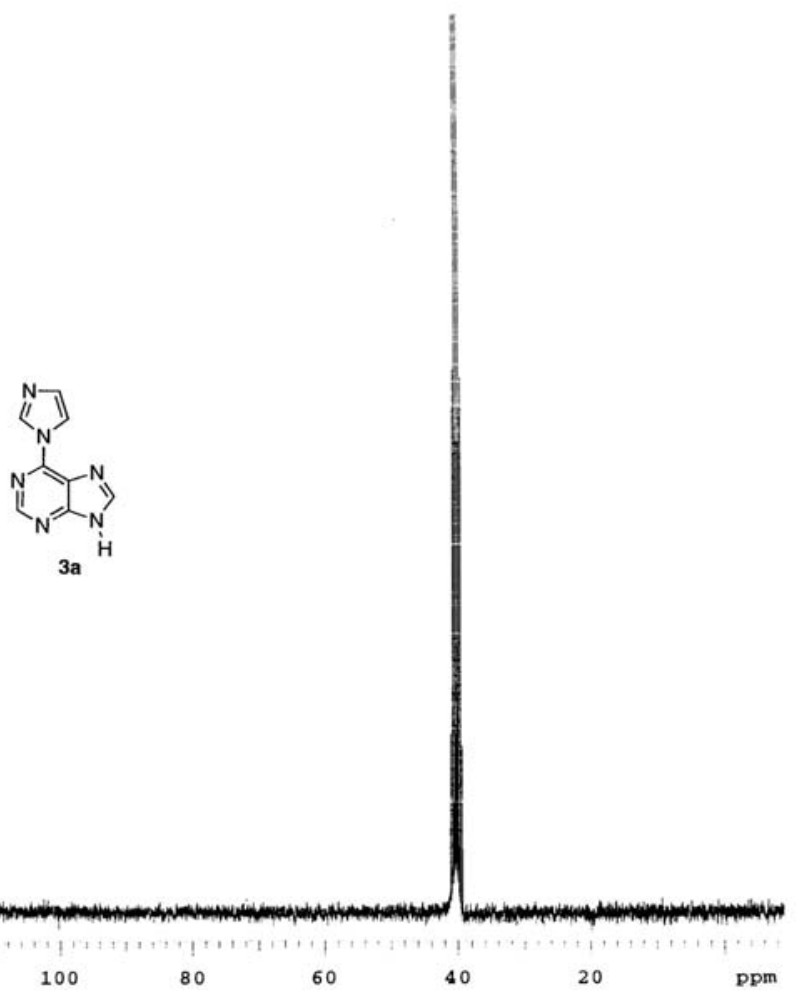




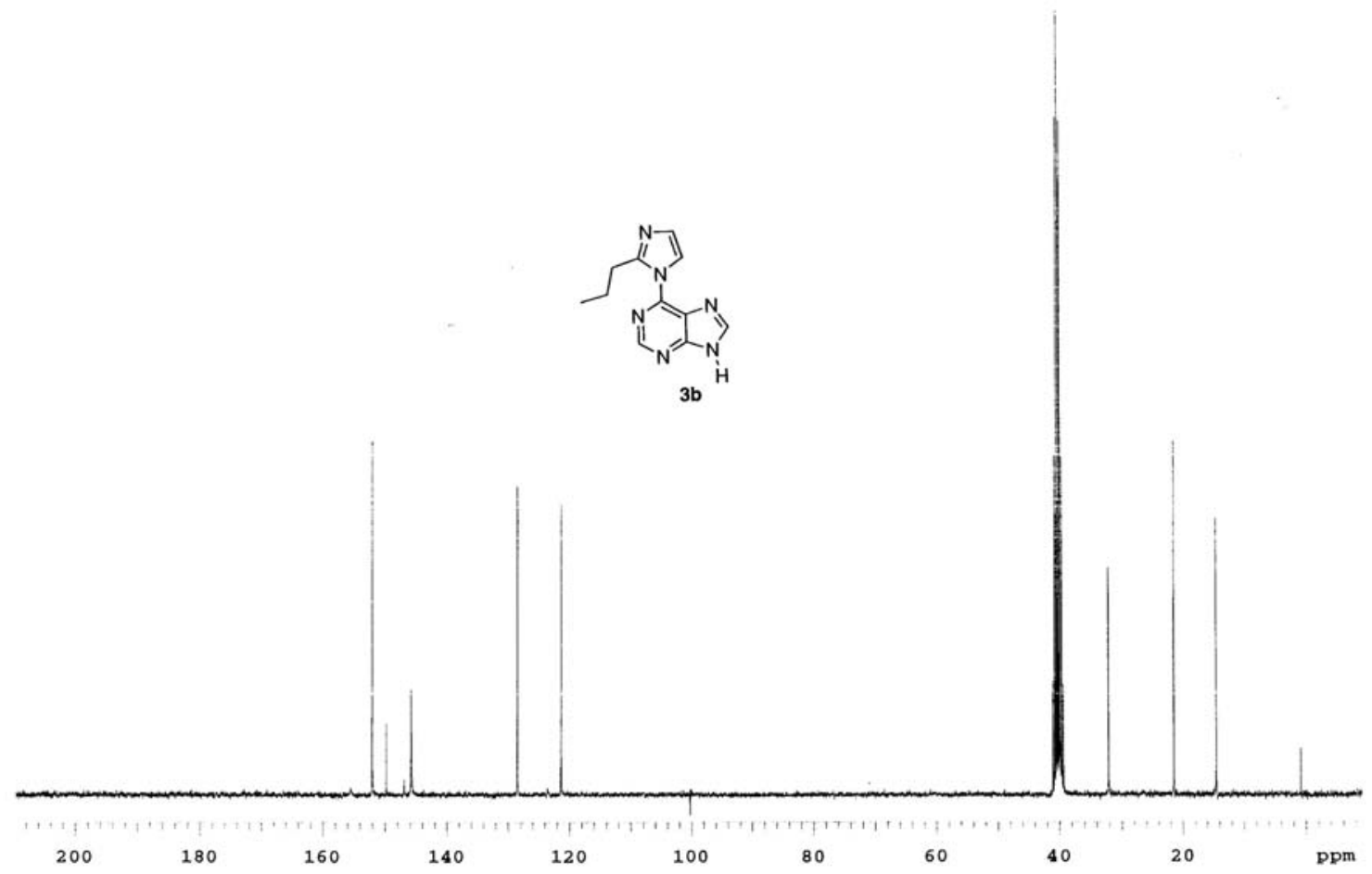




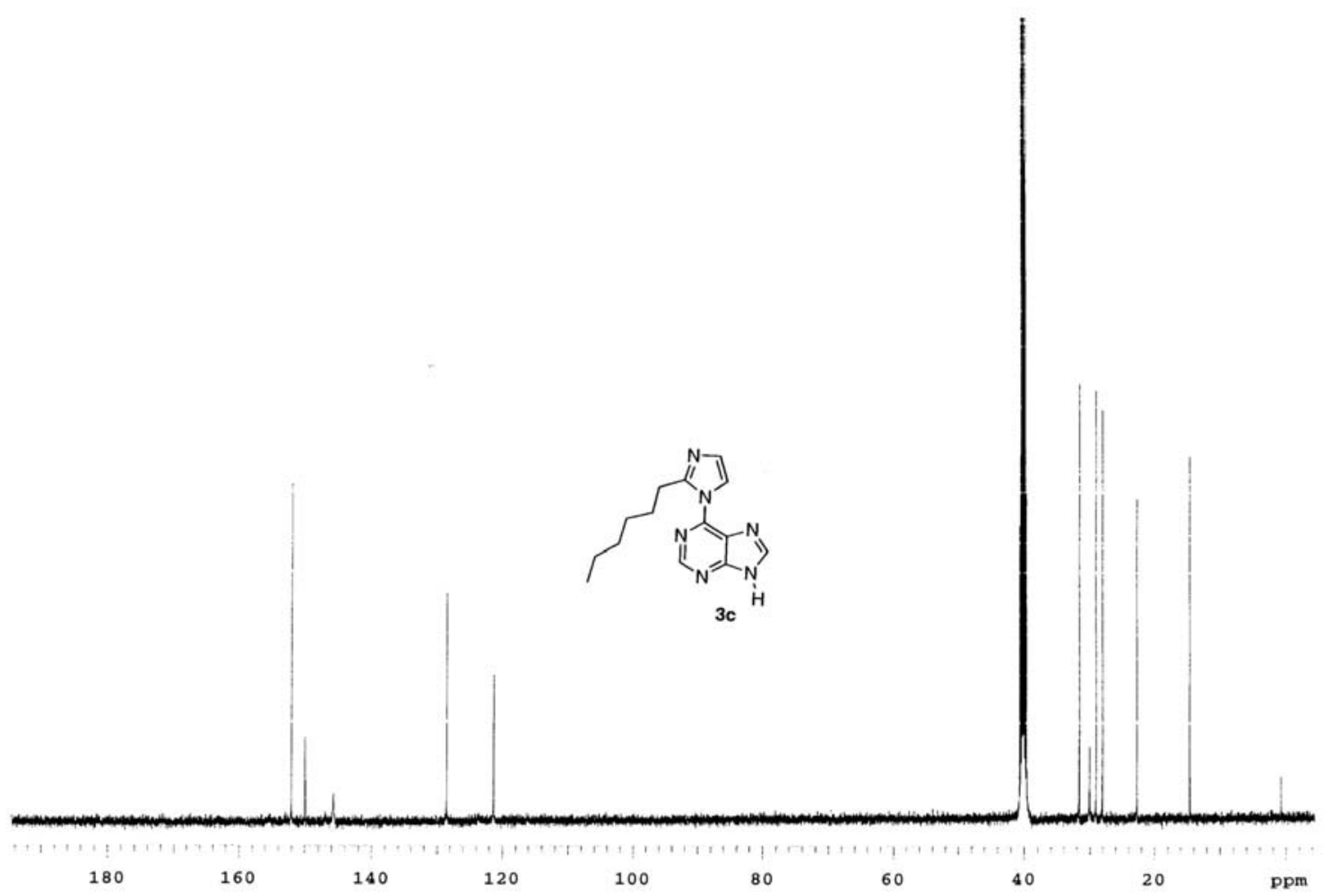




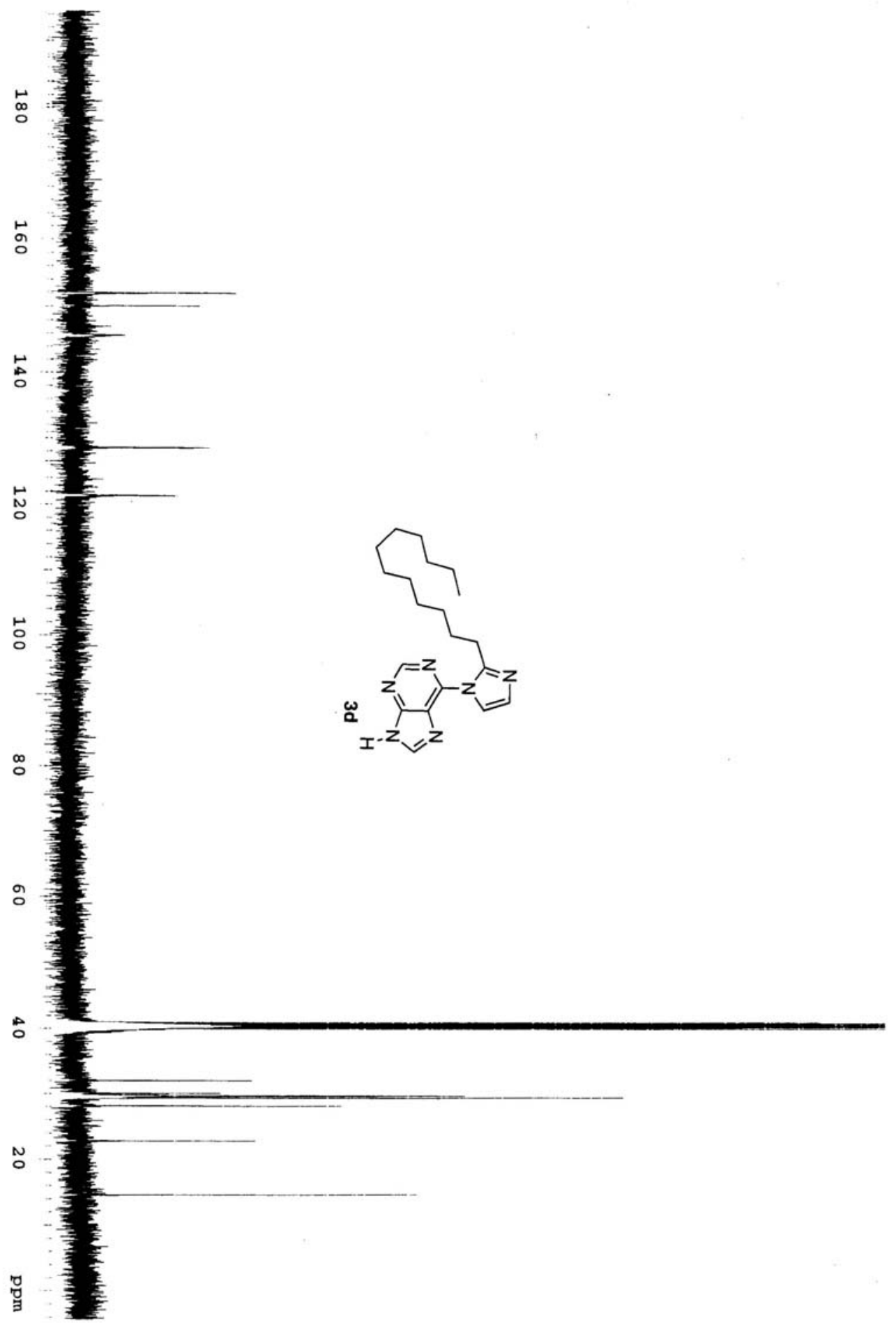




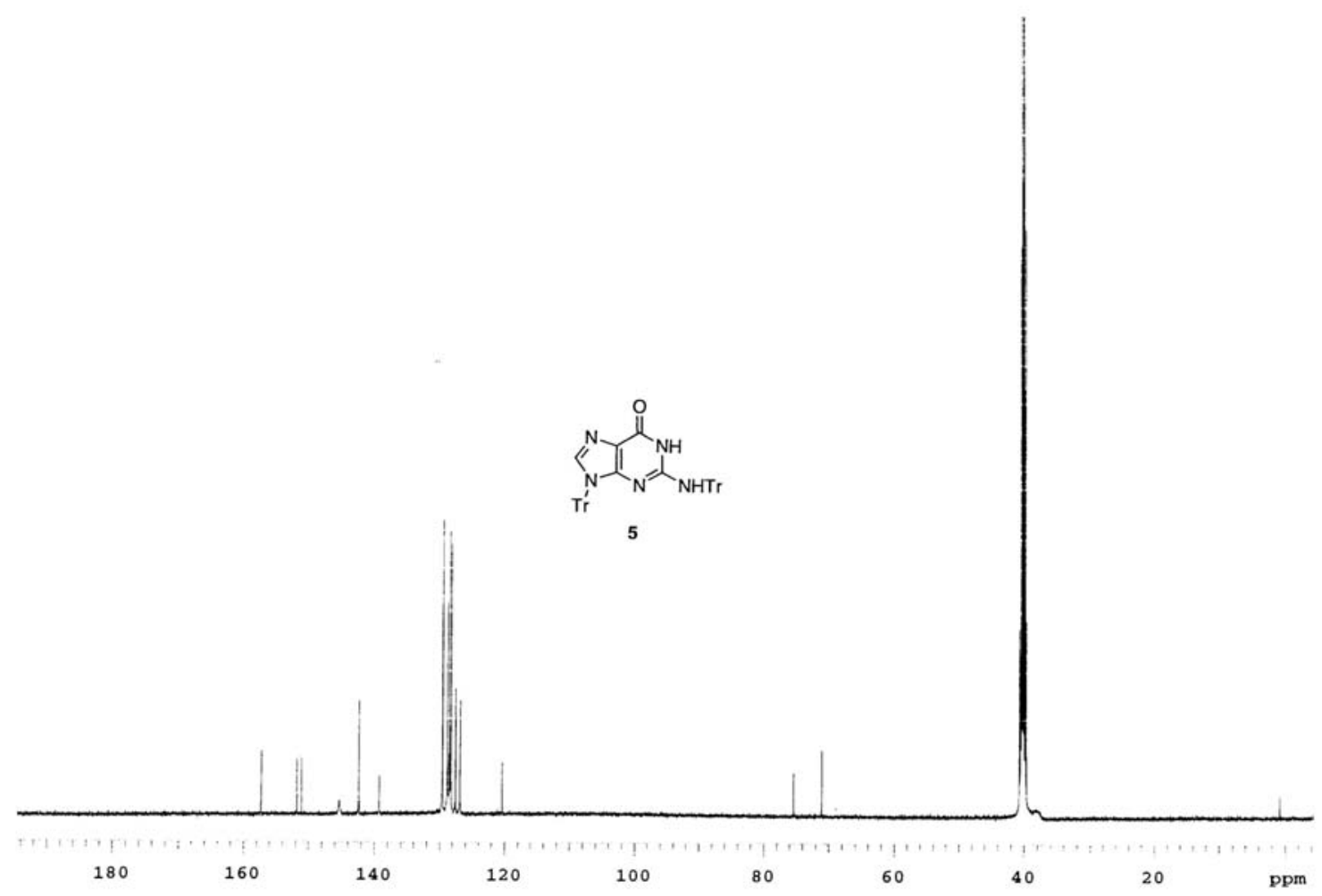




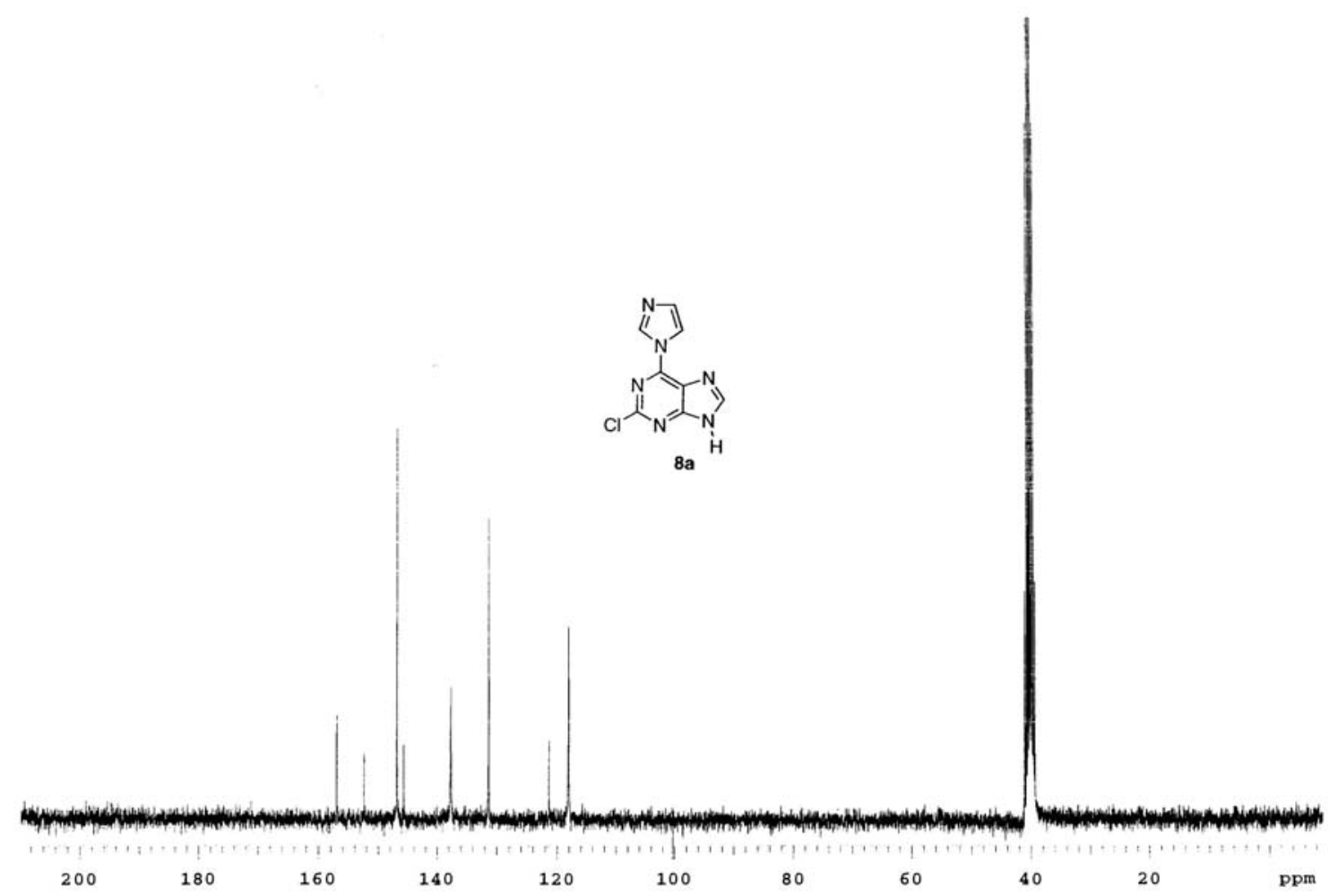




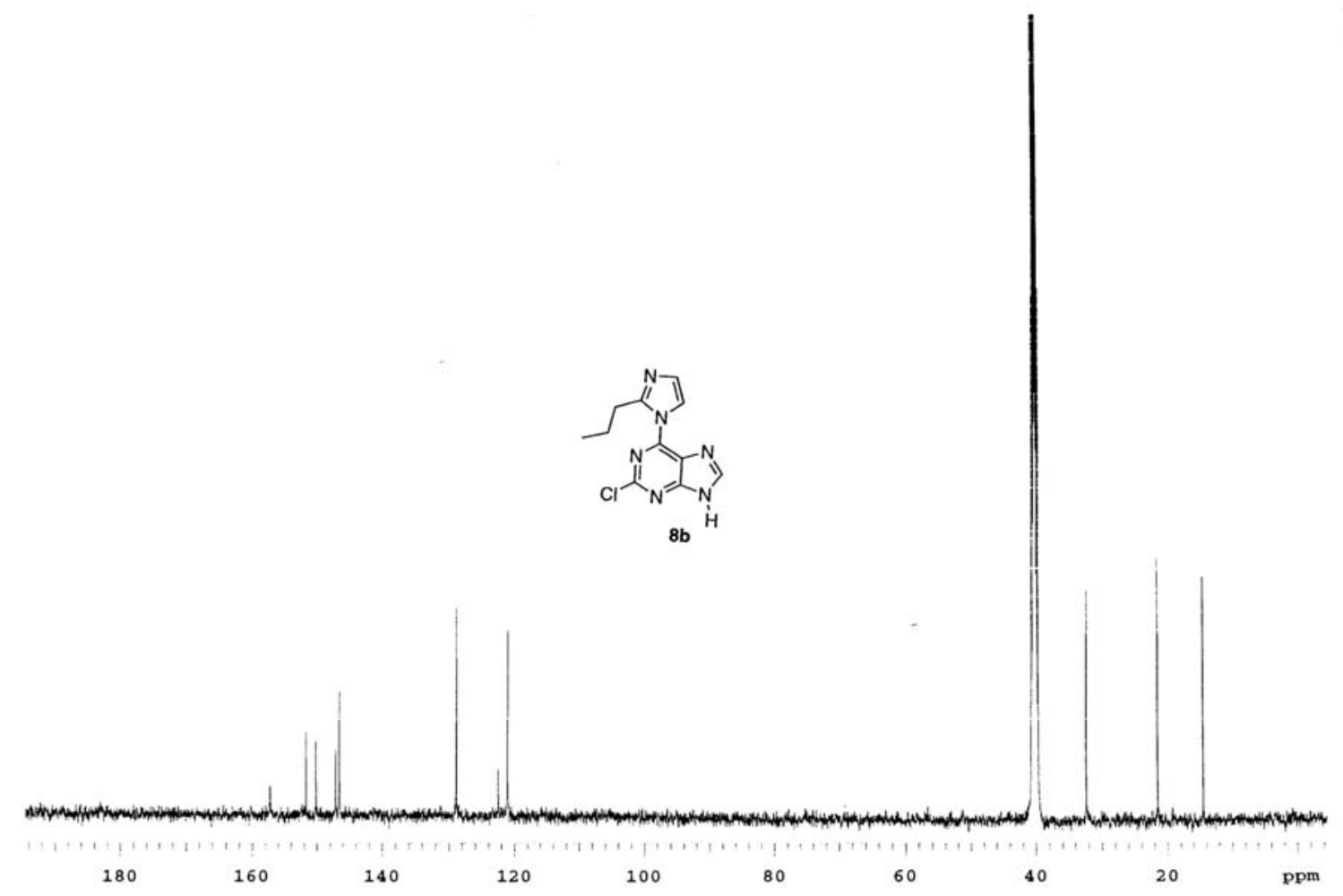




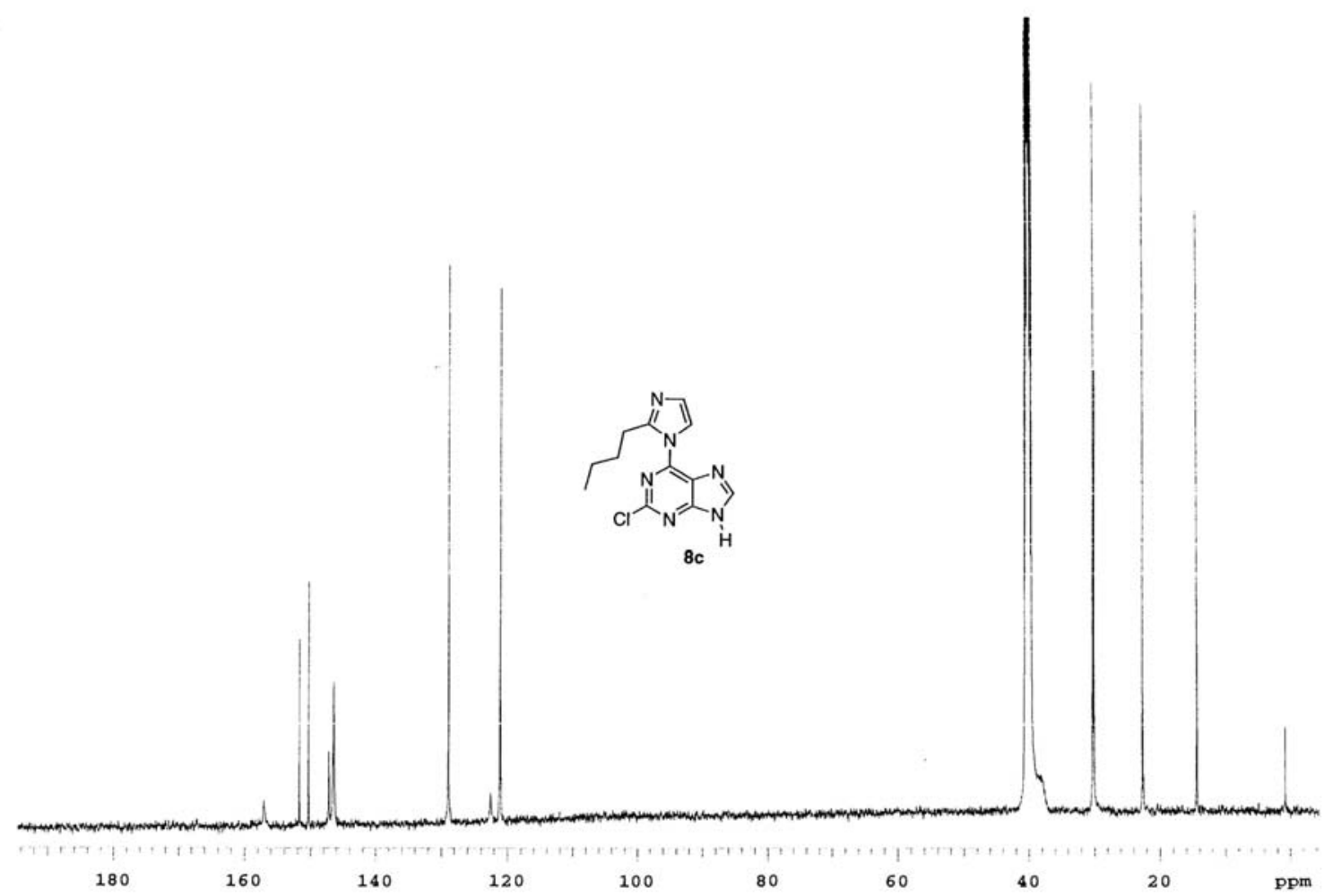




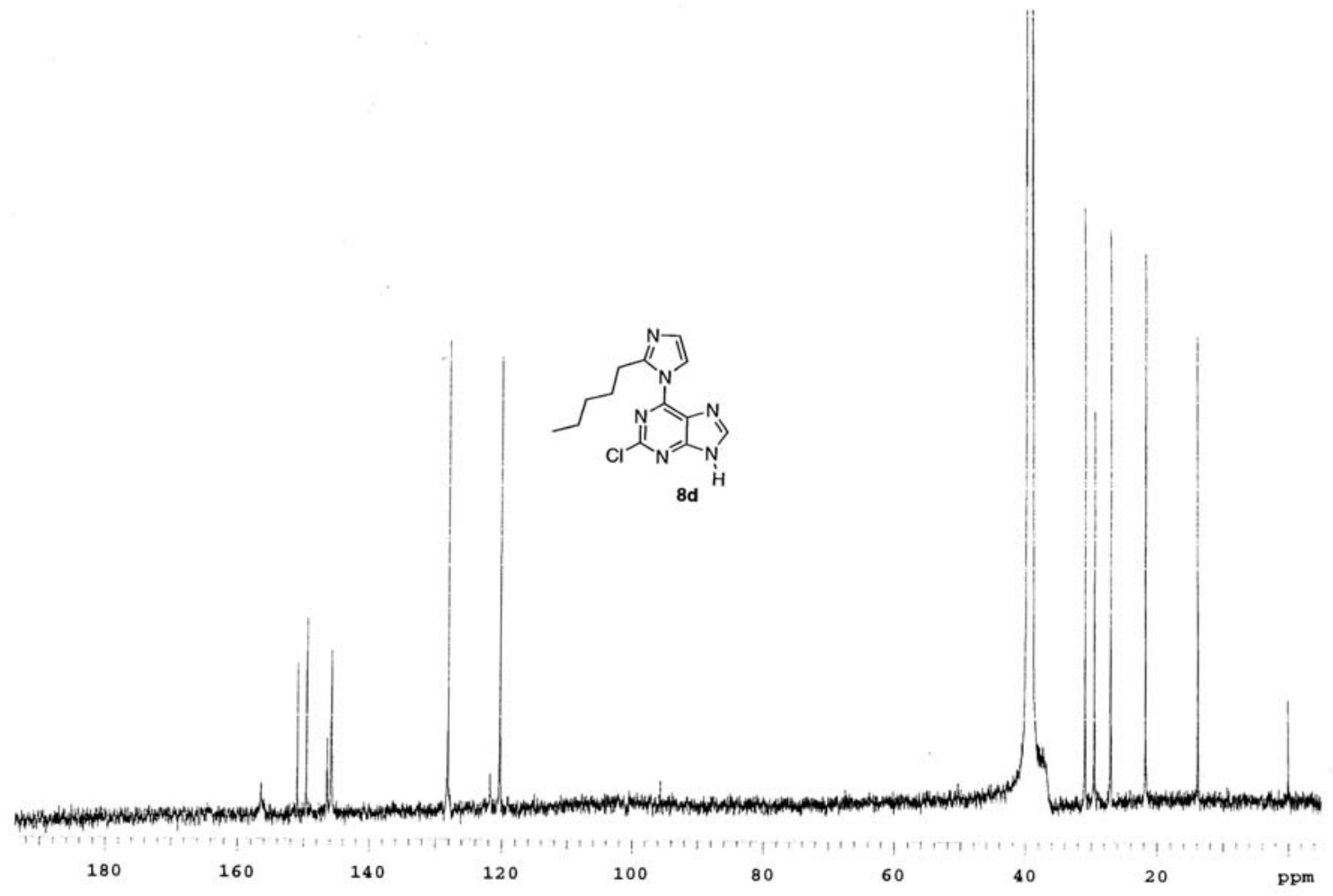




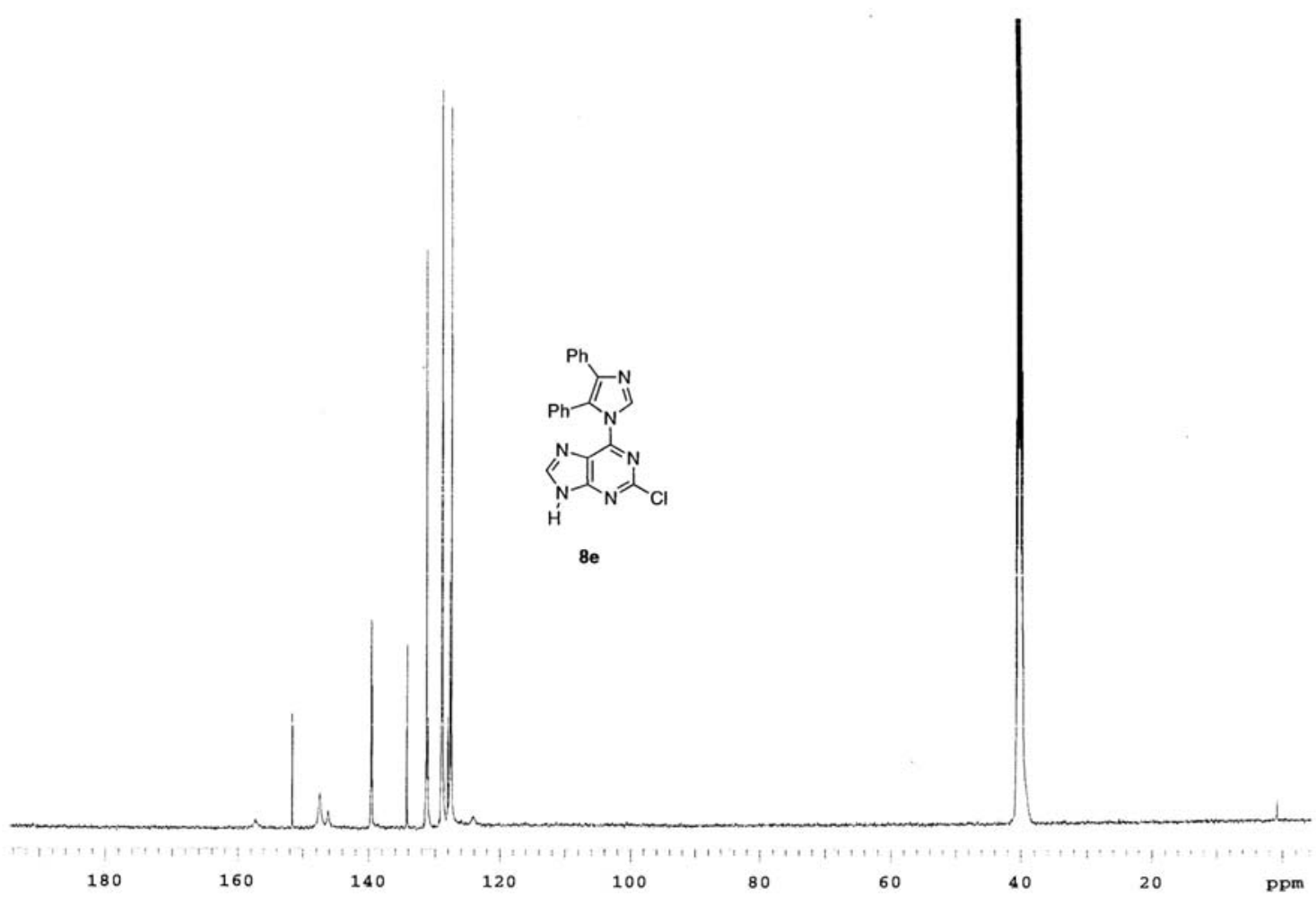




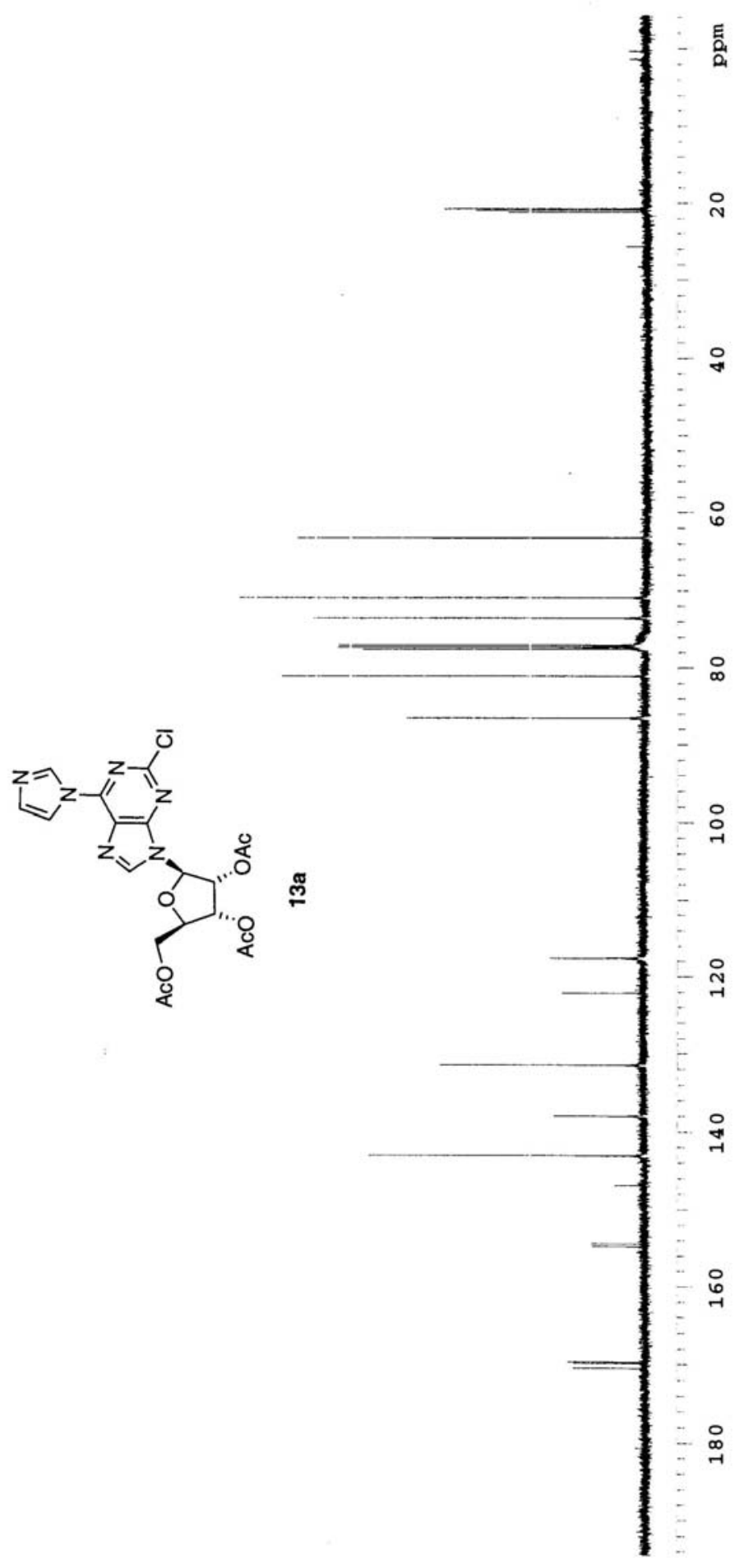


S26

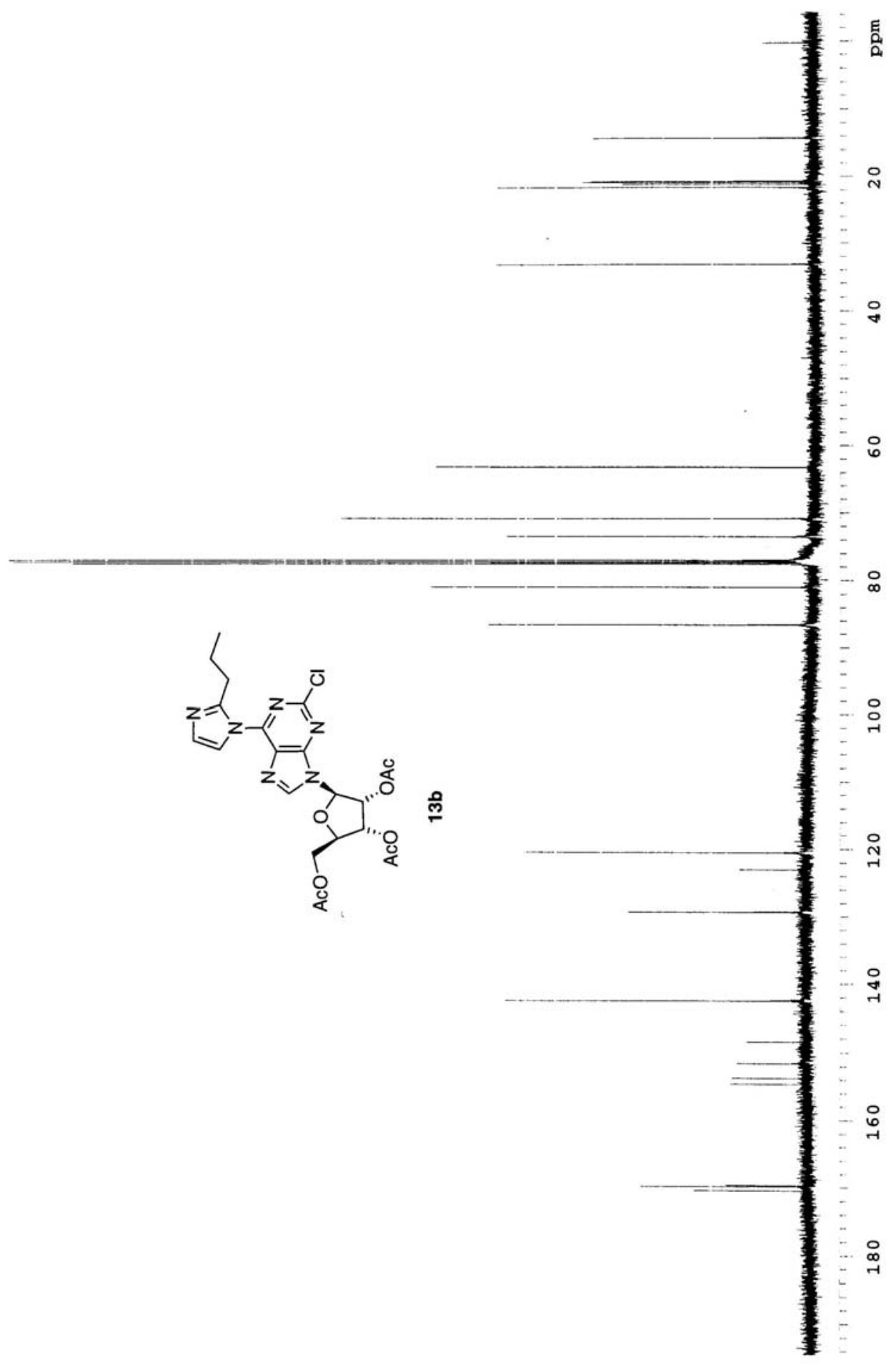




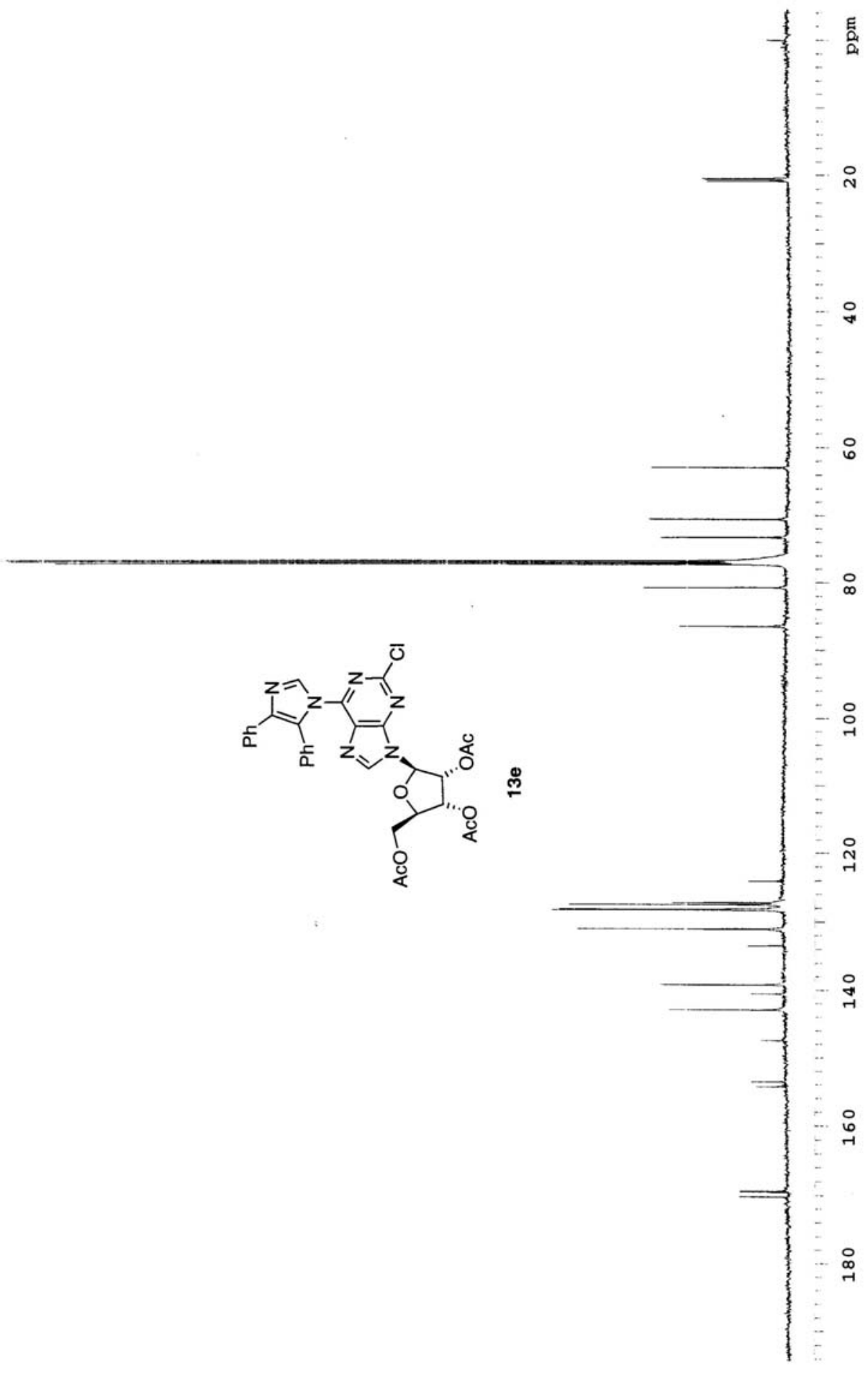




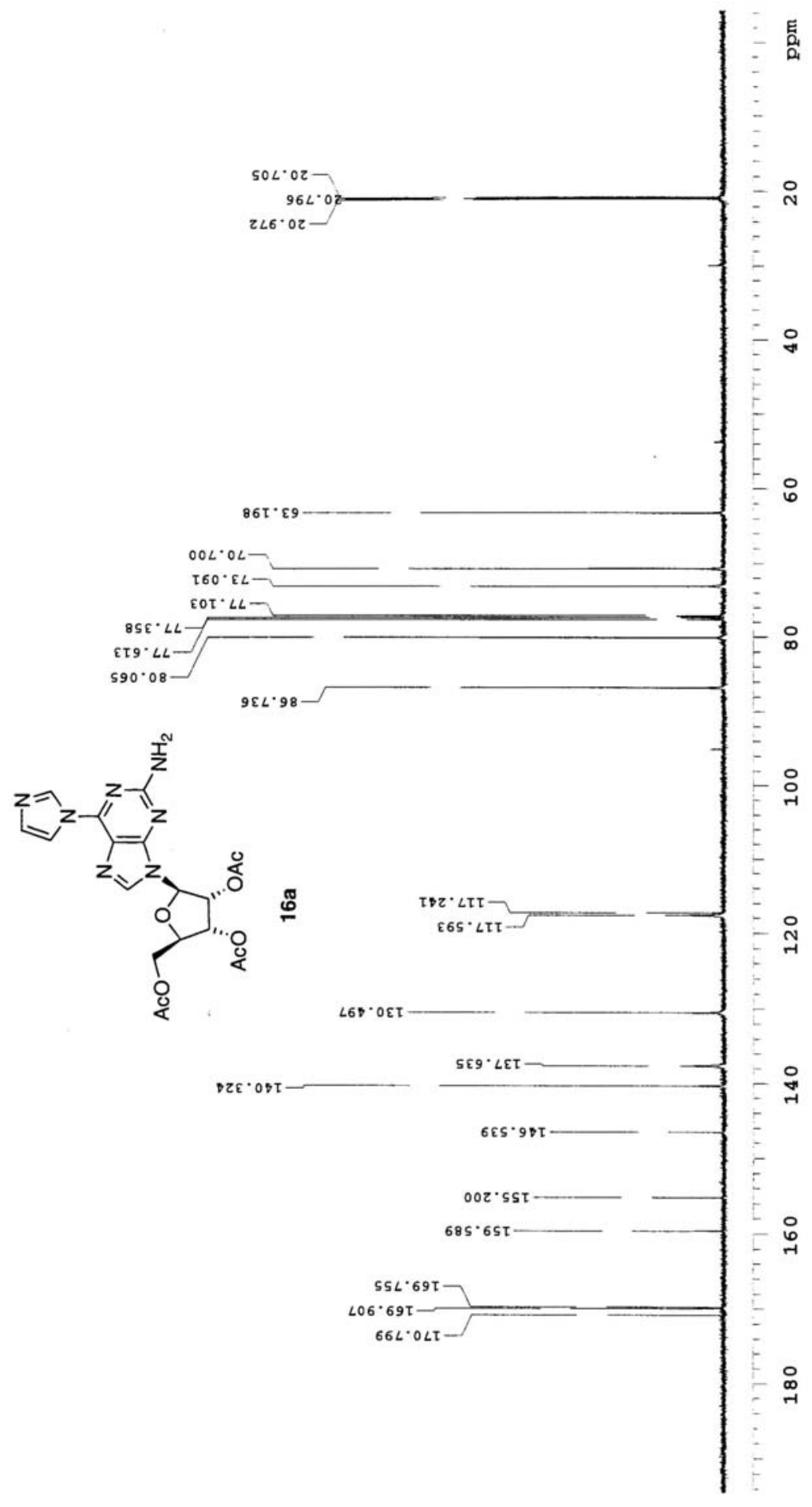

